\title{
Serial Comprehensive Geriatric Assessment in elderly head and neck cancer patients undergoing curative radiotherapy identifies evolution of multidimensional health problems and is indicative of quality of life
}

Lies Pottel ${ }^{\mathrm{a}}$, Michelle Lycke ${ }^{\mathrm{a}}$, Tom Boterberg ${ }^{\mathrm{b}}$, Hans Pottel $^{\mathrm{c}}$, Laurence Goethals ${ }^{\mathrm{a}}$, Fréderic Duprez $^{\mathrm{b}}$, Nele Van Den Noortgate ${ }^{\mathrm{d}}$, Wilfried De Neve ${ }^{\mathrm{b}}$, Sylvie Rottey ${ }^{\mathrm{e}}$, Kurt Geldhof ${ }^{\mathrm{f}}$, Véronique Buyse $^{\mathrm{g}}$, Khalil Kargar-Samani ${ }^{\mathrm{h}}$, Véronique Ghekiere ${ }^{\mathrm{i}}$ and Philip R. Debruyne ${ }^{\mathrm{a}}$

${ }^{a}$ Cancer Center, General Hospital Groeninge, Loofstraat 43, B-8500 Kortrijk, Belgium

bDepartment of Radiation Oncology, Ghent University Hospital, De Pintelaan 185, B-9000 Ghent, Belgium

${ }^{c}$ Biostatistics, Faculty of Medicine, Catholic University Leuven Kulak, Etienne Sabbelaan 53, B-8500 Kortrijk, Belgium

dDepartment of Geriatrics, Ghent University Hospital, De Pintelaan 185, B-9000 Ghent, Belgium

e Department of Medical Oncology, Ghent University Hospital, De Pintelaan 185, B-9000 Ghent, Belgium

${ }_{\mathrm{f}}^{\mathrm{f}}$ Department of Internal Medicine, Jan Yperman Hospital, Briekestraat 12, B-8900 Ypres, Belgium

'Department of Internal Medicine, General Hospital OLV Lourdes, Vijfseweg 150, B-8790 Waregem, Belgium

hDepartment of Oncology, Centre Hospitalier de Wallonie Picarde, RHMS, Chaussée de Saint-Amand 80, B7500 Tournai, Belgium

iDepartment of Geriatrics, General Hospital Groeninge, Reepkaai 4, B-8500 Kortrijk, Belgium

\begin{abstract}
Background: Almost half of head and neck $(\mathrm{H} \& \mathrm{~N})$ cancer patients is 65 years or older, however the use of comprehensive geriatric assessment (CGA) to quantify functional age in these patients has not been studied. Moreover, the search for an optimal screening tool to identify patients in need of a full CGA is still ongoing. We examined (1) a disease-specific Combined Screening Tool (CST, "VES-13+(17-G8)"), (2) the feasibility of serial CGA, and (3) its ability to indicate Quality of Life (QoL).
\end{abstract}

Methods: Patients, aged $\geq 65 \mathrm{y}$, with primary $H \& N$ cancer undergoing curative radio(chemo)therapy were evaluated with VES-13, G8, CGA, and EORTC QoL Questionnaire at baseline (W0) and during week 4 (W4) of their therapy at the General Hospital Groeninge or Ghent University Hospital. Receiver Operating Characteristic analysis was used to determine the diagnostic accuracy of the CST. Wilcoxon signed rank test and Mc Nemar's Exact test were performed for comparison of health status at both time points. Repeated measures ANOVA were applied to examine potential differences in EORTC QoL data between fit and vulnerable patients (as defined by baseline CGA).

Results: One hundred primarily male (86.0\%) patients, with a median age of 72 (range 6586), and mostly presenting with an advanced-stage cancer (69.0\%, stage III-IVb) of the larynx $(45.0 \%)$, pharynx $(34.0 \%)$, oral cavity $(15.0 \%)$, or involved neck nodes of an occult primary $(6.0 \%)$ were recruited. At W0, 71.3\%, 36.8\%, 69.0\%, and $61.2 \%$ were defined vulnerable according to CGA (cut-off $\geq 2$ positive domains), VES-13 (cut-off $\geq 3$ ), G8 (cut-off $\leq 14$ ) and 
CST (cut-off $\geq 5)$ respectively. The proportion ((Sensitivity+Specificity)/2) of the CST (79.5\%) did not significantly differ from the clinically relevant proportion of $75.0 \%$ necessary for validation. At W0, patients experienced mostly severe grade co-morbidities $(74.7 \%)$, difficulties in community functioning (50.6\%) and nutritional problems (50.6\%). Significantly more patients were considered vulnerable at W4 by CGA $(83.9 \%, \mathrm{p}<0.01)$, VES-13 (57.5\%, $\mathrm{p}<0.0001)$, and G8 (92.0\%, $\mathrm{p}<0.0001)$, deteriorating especially at the nutritional $(\mathrm{p}<0.0001)$, functional $(\mathrm{p}<0.0001)$ and emotional $(\mathrm{p}<0.01)$ level. Vulnerable patients generally reported lower QLQ functioning and higher symptom scores at both time-points, compared to fit patients. A comparable deterioration in QoL was observed in both groups through therapy.

Conclusions: We were unable to validate the CST according to the predefined hypothesis. The G8 screening tool therefore presently remains standard of care to identify patients in need of a full CGA. Our data suggest that CGA classification is indicative of QoL. Moreover, it identifies the multidimensional health problems and their evolution during radiotherapy, guiding individualized supportive care. It should therefore be integrated in the treatment approach and follow-up of elderly H\&N cancer patients. 